\title{
Jet quenching pattern at LHC in PYQUEN model
}

\author{
I.P. Lokhtin, A.V. Belyaev and A.M.Snigirev \\ D.V. Skobeltsyn Institute of Nuclear Physics, M.V. Lomonosov Moscow State University, Moscow, Russia
}

\begin{abstract}
The first LHC data on high transverse momentum hadron and dijet spectra in $\mathrm{PbPb}$ collisions at center-of-mass energy $2.76 \mathrm{TeV}$ per nucleon pair are analyzed in the frameworks of PYQUEN jet quenching model. The presented studies for the nuclear modification factor of high- $\mathrm{p}_{T}$ hadrons and the imbalance in dijet transverse energy support the supposition that the intensive wide-angular ("out-of-cone") mediuminduced partonic energy loss is seen in central $\mathrm{PbPb}$ collisions at the LHC.
\end{abstract}

\section{Introduction}

The production of high transverse momentum hadrons and jets is one of the important probes of hot quark-gluon matter (QGM) created in ultrarelativistic heavy ion collisions. The energy loss of energetic partons, so called "jet quenching", is predicted to be very different in cold nuclear matter and in hot QGM, and leads to a number of phenomena which are already seen in gold-gold collisions at RHIC $\left(\sqrt{s}_{\mathrm{NN}}=200 \mathrm{GeV}\right)$, such as suppression of high$p_{T}$ hadron spectrum, medium-modified angular correlations, azimuthal anisotropy, etc. (see, e.g., recent reviews 1, 2, 3, 4,5 and references therein). The current lead-lead collision energy at LHC, $\sqrt{s}_{\mathrm{NN}}=2.76 \mathrm{TeV}$, is a factor of $\sim 14$ larger than that in RHIC, thereby allows one to probe new frontiers of super-high temperature and (almost) net-baryon free Quantum Chromodynamics (QCD). It is expected that at such ultra-high energies the role of hard and semi-hard particle production may be significant even for the bulk properties of the created matter.

The first striking results of LHC heavy ion program are the momentum-dependent suppression of charge high$\mathrm{p}_{T}$ particle production [6] and the large imbalance in dijet transverse energy [7,8. It was supposed in 9] with simple kinematic arguments that a frequency collimation mechanism in medium-modified parton showering can account for these observables. The calculation of dijet asymmetry due to multiple scatterings of shower partons to large angles has been done in [10]. The dijet asymmetry was also computed recently with MARTINI model in [11. The decoherence of parton showering in a dense medium was considered in [12. Some numerical predictions for jet broadening in $\mathrm{PbPb}$ collisions at the LHC can be found in 13 . In present paper, the jet quenching pattern at the LHC is analyzed in the frameworks of PYQUEN partonic energy loss model [14], and the related interpretation of the data is discussed.

\section{PYQUEN partonic energy loss model}

A number of Monte-Carlo models to combine a perturbative final state parton shower with QCD-medium effects and simulate the jet quenching in various approaches have been developed in recent years $14,15,16,17,18,19$. Our model PYQUEN (PYthia QUENched) 14 was constructed as a modification of the jet event obtained with the generator of hadron-hadron interactions PYTHIA_6.4 [20]. The details of PYQUEN physics model and simulation procedure can be found in [14], the main features of the model being listed only very briefly below.

The approach to the description of medium-induced multiple scattering of hard partons in PYQUEN is based on the accumulated energy loss via the gluon radiation being associated with each parton scattering in the expanding quark-gluon fluid and includes the interference effect (for the emission of gluons with a finite formation time) using the modified radiation spectrum $d E / d x$ as a function of decreasing temperature $T$. The model takes into account the radiative and collisional energy loss of hard partons in longitudinally expanding quark-gluon fluid, as well as a realistic nuclear geometry. The radiative energy loss is treated in the frameworks of BDMS model [21,22] with the simple generalization to a massive quark case using the "dead-cone" approximation 23. The collisional energy loss due to elastic scatterings is calculated in the high-momentum transfer limit 24,25,26]. The strength of the energy loss in PYQUEN is determined mainly by the initial maximal temperature $T_{0}^{\max }$ of hot matter in central $\mathrm{PbPb}$ collisions (i.e. an initial temperature in the center of nuclear overlapping area at mid-rapidity). The energy loss depends also on the proper time $\tau_{0}$ of QGM formation and the number $N_{f}$ of active flavors in the medium. The parameter values $\tau_{0}=0.1 \mathrm{fm} / c$ and $N_{f}=0$ (gluondominated plasma) are used for the simulations presented.

Another important ingredient of the model is the angular spectrum of medium-induced radiation. Since the detailed treatment of this is rather sophisticated, the simple parametrizations of the gluon distribution over the 
emission angle $\theta$ are used. There are two main options in PYQUEN. The first one is the "small-angular" radiation,

$$
\frac{d N^{g}}{d \theta} \propto \sin \theta \exp \left(-\frac{\left(\theta-\theta_{0}\right)^{2}}{2 \theta_{0}^{2}}\right),
$$

where $\theta_{0} \sim 5^{0}$ is the typical angle of the coherent gluon radiation as estimated in 27 . The second one is the "wideangular" radiation,

$$
\frac{d N^{g}}{d \theta} \propto 1 / \theta
$$

which is similar to the angular spectrum of parton showering in a vacuum without coherent effects 28. In this paper, in order to illustrate the sensitivity of experimental observables to various jet quenching scenarios, the third (rather extreme) option "extra wide-angular" radiation,

$$
\frac{d N^{g}}{d \theta} \propto 1 / \sqrt{\theta}
$$

is also considered. One can assume that the physical meaning of (extra) wide-angular radiation could be the presence of intensive secondary rescatterings of in-medium emitted gluons 10. The collisional energy loss is considered in PYQUEN as "out-of-cone" loss (i.e. an energy is "absorbed" by the medium, because the major part of "thermal" particles knocked out of the dense matter by elastic rescatterings fly outside a typical jet cone [27]).

The event-by-event simulation procedure in PYQUEN includes the following steps.

- The generation of initial parton spectra with PYTHIA and production vertexes at the given impact parameter.

- The rescattering-by-rescattering simulation of the parton path in a dense zone and its radiative and collisional energy loss.

- The final hadronization according to the Lund string model for hard partons and in-medium emitted gluons.

In presented studies, the effect of nuclear shadowing on parton distribution functions, calculating the nuclear modification factor $R_{\mathrm{AA}}$ for charged particles at $p_{T}>$ $10 \mathrm{GeV}$, is taken into account. The procedure is similar to one applied in HYDJET and HYDJET ++ event generators 29]. It uses the impact parameter dependent parametrization obtained in the frameworks of GlauberGribov theory 30. The nuclear shadowing correction factor $S\left(r_{1}, r_{2}, x_{1}, x_{2}, Q^{2}\right)$ depends on the kinematic variables of incoming hard partons (the momentum fractions $x_{1,2}$ of the initial partons from the incoming nuclei and the momentum scale $Q^{2}$ of the hard scattering), and on the transverse coordinates $r_{1,2}$ of the partons in their respective nuclei. This initial state effect reduces the number of partons in the incoming hadronic wave-function of both the nuclei and thus reduces the yield of high- $\mathrm{p}_{T}$ hadrons from jet production (in addition to the medium-induced partonic energy loss).

\section{Numerical results}

PYQUEN partonic energy loss model was applied to calculate the nuclear modification factor of charged hadrons and the imbalance in dijet transverse energy in central $\mathrm{PbPb}$ collisions at $\sqrt{s}_{\mathrm{NN}}=2.76 \mathrm{TeV}$ for various scenarios of medium-induced radiation. The numerical results were compared with the LHC data applying the same kinematic cuts as in the experiments.

\subsection{Nuclear modification factor}

The nuclear modification factor $R_{\mathrm{AA}}$ is defined as a ratio of particle yields in AA and pp collisions normalized on the number of binary nucleon-nucleon sub-collisions $\left\langle N_{\text {coll }}\right\rangle$ :

$$
R_{\mathrm{AA}}\left(p_{T}\right)=\frac{d^{2} N^{\mathrm{AA}} / d \eta d p_{T}}{\left\langle N_{\mathrm{coll}}\right\rangle d^{2} N^{\mathrm{AA}} / d \eta d p_{T}},
$$

where $p_{T}$ is the transverse momentum and $\eta$ is the pseudorapidity. In the absence of nuclear effects (in initial or final states) for high $p_{T}$ it should be $R_{\mathrm{AA}}=1$. Figure 1 shows the calculated nuclear modification factor $R_{\mathrm{AA}}$ for charged hadrons $(|\eta|<0.8)$ as compared with ALICE data [6] in $5 \%$ of most central $\mathrm{PbPb}$ collisions $\left(T_{0}^{\max }=0.85 \mathrm{GeV}\right.$ for the left plot and $1 \mathrm{GeV}$ for the right plot). Three options for the angular spectrum of in-medium gluon radiation (11), (2) and (3) were considered, collisional energy loss and nuclear shadowing being also taken into account. Higher initial temperatures result in more suppression of high$\mathrm{p}_{T}$ hadrons, while a wider angular radiation results in a stronger dependence of $R_{\mathrm{AA}}$ on $p_{T}$. The PYQUEN scenarios with $T_{0}^{\max }=1 \mathrm{GeV}$ and wide-angular radiation (2) or (3) seem closer to the data than other options. However even in this case, the simulated $p_{T}$-dependence of $R_{\mathrm{AA}}$ looks weaker than the tendency of the ALICE data. The large systematic uncertainties of the data (due to the absence of pp data at the same energy) still complicate a rigid quantitative interpretation of this observation. But if such discrepancy would be confirmed in the future with higher statistics and reference pp data at $\sqrt{s}=2.76 \mathrm{TeV}$, in our opinion it could indicate that the real energy dependence of partonic energy loss $\Delta E$ is weaker than the expected one. It is worth to remind here that the energy dependence of BDMS radiative loss [21,22] (the dominant mechanism of medium-induced energy loss in PYQUEN) is between $\Delta E \propto \ln E$ and $\Delta E \propto \sqrt{E}$ (depending on the energy of the radiated gluon and on the properties of the medium).

Let us discuss some other model-dependent uncertainties of the results obtained. Excluding the collisional loss from the simulations increases $R_{\mathrm{AA}}$ by $\sim 15 \div 20 \%$ for $p_{T}=10 \div 20 \mathrm{GeV} / c$. Excluding the nuclear shadowing increases $R_{\mathrm{AA}}$ by $\sim 30 \div 20 \%$ for the same kinematic range and weakens its $p_{T}$-dependence. The choice of the medium formation time $\tau_{0}$ within its reasonable range has rather moderate influence on the strength of medium-induced partonic energy loss. We have found that increasing $\tau_{0}$ by factor 2 (at fixed $T_{0}^{\max }=1 \mathrm{GeV}$ ) reduces $R_{\mathrm{AA}}$ by 

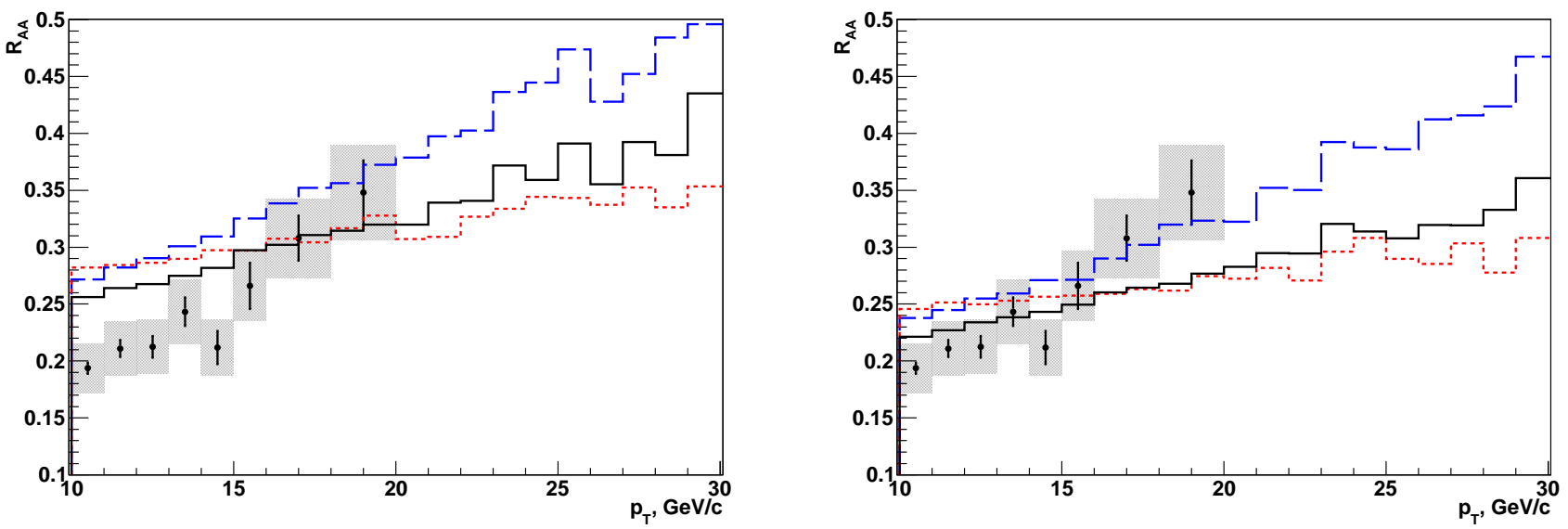

Fig. 1. The nuclear modification factor $R_{\mathrm{AA}}$ for charged hadrons in $5 \%$ of most central $\mathrm{PbPb}$ collisions at $\sqrt{s}_{\mathrm{NN}}=2.76 \mathrm{TeV}$. The points are ALICE data [6] (the error bars show the statistical uncertainties, the boxes show the systematic errors), the histograms are simulated PYQUEN events. The dotted, solid and dashed histograms correspond to the parametrizations of the radiation angular spectrum (1), (2) and (3) respectively. The initial maximal temperature of quark-gluon matter $T_{0}^{\text {max }}=0.85$ $\mathrm{GeV}$ (left) and $1 \mathrm{GeV}$ (right).
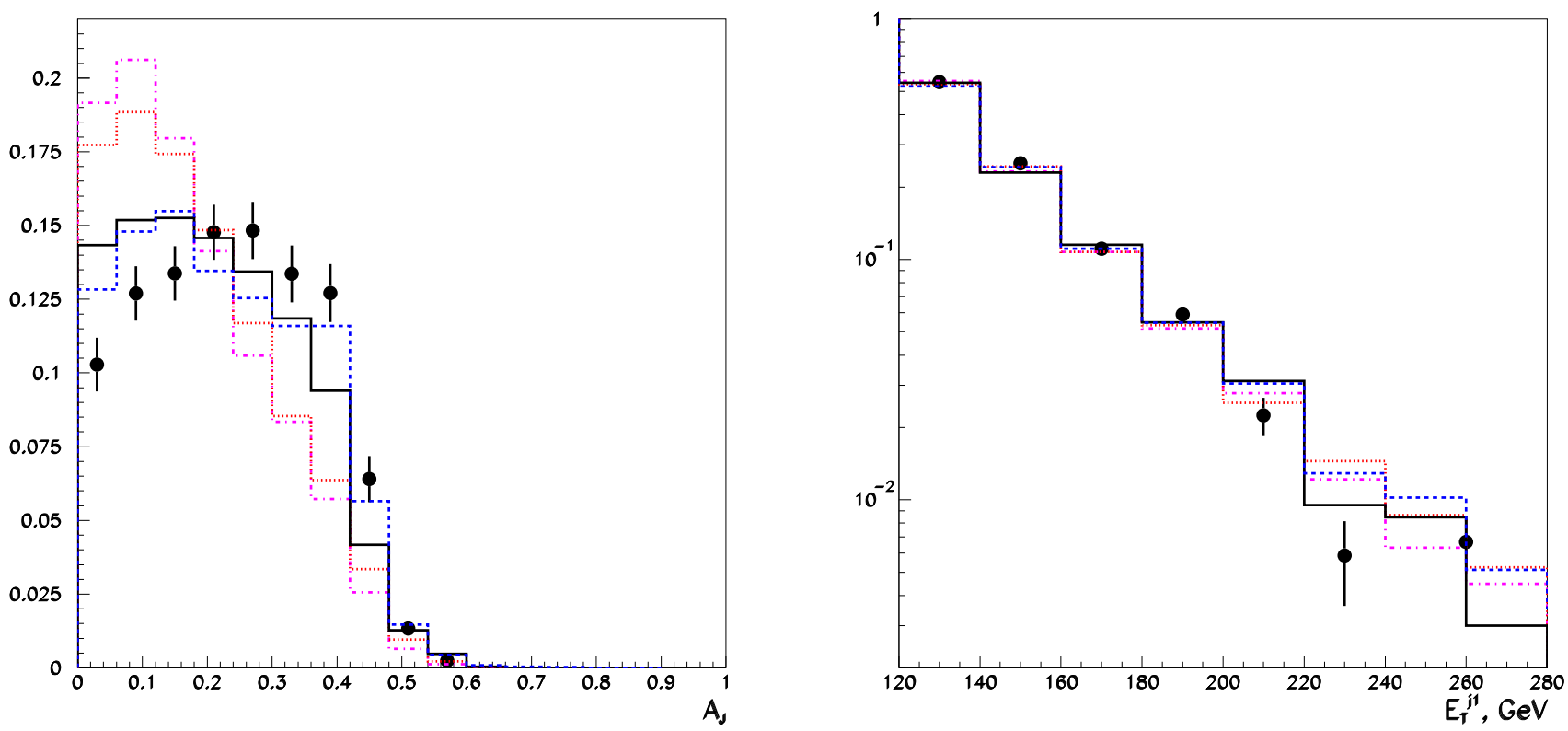

Fig. 2. The event fraction of dijet events with the asymmetry ratio $A_{J}$ (left) and with the leading jet energy $E_{T}^{j 1}$ (right) in $10 \%$ of most central $\mathrm{PbPb}$ collisions at $\sqrt{s}_{\mathrm{NN}}=2.76 \mathrm{TeV}$. The points are CMS data 8] (the error bars show the statistical uncertainties), the histograms are simulated PYTHIA (dash-dotted) and PYQUEN (other styles) events. The dotted, solid and dashed histograms correspond to the parametrizations of the radiation angular spectrum (1), (2) and (3) respectively. The initial maximal temperature of quark-gluon matter $T_{0}^{\max }=1 \mathrm{GeV}$.

$\sim 13 \%$ without changing its $p_{T}$-dependence. Note that the jet quenching effect gets stronger at larger $\tau_{0}$ due to slower cooling of the matter, which implies a jet parton spending more time in the hottest regions of the medium. As a result the rescattering intensity increases (it starts little later for larger $\tau_{0}$, but this factor is much less significant). Fixing $\tau_{0}=0.2 \mathrm{fm} / c$ and decreasing $T_{0}^{\max }$ to $0.85 \mathrm{GeV}$ allow us to get the similar results for $R_{\mathrm{AA}}\left(p_{T}\right)$ as shown in Figure 1 (right). The jet quenching effect is also sensitive to the space-time evolution of a dense matter. For calculations presented, the medium was treated as a boost-invariant longitudinally expanding perfect quark-gluon fluid (wellknown scaling solution obtained originally by Bjorken [31, $\left.T(\tau)=T_{0}\left(\tau_{0} / \tau\right)^{1 / 3}\right)$. In principle, other scenarios of QGM space-time evolution for PYQUEN are also envisaged [14, 26]. For example, the presence of fluid viscosity slows down 
the cooling rate, i.e., in fact the effective temperature of the medium gets higher as compared with the perfect QGM case. On the other hand, the influence of the transverse expansion on the intensity of parton rescattering is inessential for high initial temperatures $T_{0}^{\max } \gg T_{c}$ ( $T_{c} \sim 200 \mathrm{MeV}$ is the critical temperature).

\subsection{Dijet transverse energy imbalance}

Dijet asymmetry ratio $A_{J}$ is defined [7,8, as a difference between transverse energies of two jets $j 1$ and $j 2$ normalized on its sum:

$$
A_{J}=\frac{E_{T}^{j 1}-E_{T}^{j 2}}{E_{T}^{j 1}+E_{T}^{j 2}} .
$$

In order to define calorimetric jets, we use the toy jet finding model. The jet energy was determined as the total transverse energy of the final particles collected around the direction of the leading particle inside the cone of radius $R=\sqrt{\Delta \eta^{2}+\Delta \varphi^{2}}=0.5(\eta$ and $\varphi$ are the pseudorapidity and the azimuthal angle respectively). Then the final jet energy was smeared using Gaussian distribution with $\sigma$ proportional to the square root of a "true" jet energy. The same kinematic cuts as in the CMS analysis [8] were applied: $\left|\eta^{j 1,2}\right|<2,\left|\varphi^{j 1}-\varphi^{j 2}\right|>2.1, E_{T}^{j 1}>120 \mathrm{GeV}$, $E_{T}^{j 2}>50 \mathrm{GeV}$. Note that we compare our simulations with the CMS data having harder cuts on minimum jet energy than ATLAS data, in order to reduce complications facing jet reconstruction in high multiplicity background [32,11.

Figure 2 (left) shows the calculated fraction of dijet events with the asymmetry ratio $A_{J}$ for three options of the angular spectrum of in-medium gluon radiation (11), (2) and (3) respectively. The choice of main parameters $\left(T_{0}^{\max }=1 \mathrm{GeV}, \tau_{0}=0.1 \mathrm{fm} / c\right)$ was fixed from fitting the LHC data on $R_{\mathrm{AA}}\left(p_{T}\right)$ discussed in previous sub-section. The collisional energy loss is taken into account, the nuclear shadowing effect being negligible for such high $\mathrm{E}_{T}$ jets. PYQUEN results are compared with CMS data 8 for $10 \%$ of most central $\mathrm{PbPb}$ collisions and with PYTHIA simulations (in fact, PYQUEN without medium effects). The option with the small-angular radiative energy loss (11) does not result in any additional dijet asymmetry as compared with PYTHIA (small additional asymmetry seen for dotted histogram is due to the collisional loss). Indeed, in this case the bulk of in-medium emitted gluons fly close to the parent parton direction and still belong to the jet. Therefore, the small-angular medium-induced radiation softens particle energy distributions inside the jet and increases the multiplicity of secondary particles, but does not affect the total jet energy [27. On the other hand, the wide-angular radiation (2) and (3) generates the significant dijet asymmetry due to the substantial fraction of "out-of-cone" jet energy loss. We would not expect the perfect agreement of PYQUEN simulations with the data using our oversimplified treatment of jet finding (without taking into account realistic detector effects, jet reconstruction details and high multiplicity background fluctuations), but at least qualitative reproducing of the dijet asymmetry can be achieved with the wide-angular radiative and collisional partonic energy loss. This interpretation is in the agreement with the observation of CMS collaboration that the dijet momentum balance is recovered when integrating low transverse momentum particles distributed over a wide angular range relative to the direction of the away-side jet 8 .

Figure 2 (right) represents the distribution over the leading jet energy $E_{T}^{j 1}$. This distribution seems quite stable. It does not depend significantly on jet quenching scenario, and is similar for PYQUEN, PYTHIA and CMS data. It confirms that the considered dijet configurations are dominated by a "surface emission", when the dijet production vertex is close to the surface of the nuclear overlapping area. In this case one partonic jet escapes from the dense zone almost without interactions and produces the leading hadronic jet, while a second partonic jet is affected by medium-induced multiple interactions and produces the "quenched" hadronic jet.

Note that the absolute number of dijet events (having leading jet with $E_{T}^{j 1}>120 \mathrm{GeV}$ and away-side jet with $E_{T}^{j 2}>50 \mathrm{GeV}$ ) is suppressed in PYQUEN as compared with PYTHIA due to reducing the number of away-side jets observed above the threshold $50 \mathrm{GeV}$ (it corresponds to increasing the "monojet" events). The dijet suppression factor depends on the radiation angular spectrum and is estimated as $0.53,0.36$ and 0.29 for PYQUEN options (1), (2) and (3) respectively.

\section{Conclusions}

The first LHC data on high- $\mathrm{p}_{T}$ hadron and dijet spectra in $\mathrm{PbPb}$ collisions at $\sqrt{s}_{\mathrm{NN}}=2.76 \mathrm{TeV}$ are analyzed in the frameworks of PYQUEN jet quenching model. The presented studies for the nuclear modification factor of high$\mathrm{p}_{T}$ hadrons and the imbalance in dijet transverse energy support the supposition that the intensive wide-angular ("out-of-cone") medium-induced partonic energy loss is seen in central $\mathrm{PbPb}$ collisions at the LHC. This interpretation is in the agreement with the recent observation of CMS collaboration that the dijet momentum balance is recovered when integrating low transverse momentum particles distributed over a wide angular range relative to the direction of the away-side jet.

However, there are still some complications for a more quantitative interpretation of the jet quenching pattern at the LHC. The measured by ALICE nuclear modification factor $R_{\mathrm{AA}}$ is affected by large systematic uncertainties due to the absence of pp data at the same energy. Moreover, the contribution of nuclear shadowing to $R_{\mathrm{AA}}$ seems visible at least up to $p_{T} \sim 25 \mathrm{GeV} / c$ and should be taken into account. Concerning ATLAS and CMS data on the dijet asymmetry, simple generator level studies are likely not enough to quantify this observable accurately (jet finding details and high multiplicity background fluctuations may be important). 
Discussions with V.L. Korotkikh, L.V. Malinina, L.I. Sarycheva, S.V. Petrushanko and B. Wyslouch are gratefully acknowledged. This work was supported by Russian Ministry for Education and Science (contract 02.740.11.0244), Grant of President of Russian Federation (No 4142.2010.2), Russian Foundation for Basic Research (grant 10-02-93118) and Dynasty Foundation.

\section{References}

1. D. d'Enterria, arXiv:0902.2011 [nucl-ex]

2. U.A. Wiedemann, arXiv:0908.2306 [hep-ph]

3. A. Accardi, F. Arleo, W.K. Brooks, D. d'Enterria, V. Muccifora, Riv. Nuovo Cim. 32, (2010) 439

4. A. Majumder, M. Van Leeuwen, arXiv:1002.2206 [hep-ph]

5. I.M. Dremin, A.V. Leonidov, Phys. Usp 53, (2011) 1123

6. K. Aamodt, et al. (ALICE Collaboration), Phys. Lett. B 696, (2011) 30

7. G. Aad, et al. (ATLAS Collaboration), Phys. Rev. Lett. 105, (2010) 252303

8. S. Chatrchyan, et al. (CMS Collaboration), arXiv:1102.1957 [nucl-ex]

9. J. Casalderrey-Solana, J.G. Milhano, U.A. Wiedemann, J. Phys. G 8, (2011) 035006

10. G.-Y. Qin, B. Muller, arXiv:1012.5280 [hep-ph]

11. C. Young, B. Schenke, S. Jeon, C. Gale, arXiv:1103.5769 [nucl-th]

12. Y. Mehtar-Tani, C.A. Salgado, K. Tywoniuk, arXiv:1102.4317 [hep-ph]

13. I. Vitev, S. Wicks, B.-W. Zhang, JHEP 0811, (2008) 093

14. I.P. Lokhtin, A.M. Snigirev, Eur. Phys. J. C 45, (2006) 211

15. K. Zapp, G. Ingelman, J. Rathsman, J. Stachel, U.A. Wiedemann, Eur. Phys. J. C 60, (2009) 617

16. K. Zapp, J. Stachel, U.A. Wiedemann, Phys. Rev. Lett. 103, (2009) 152302

17. N. Armesto, L. Cunqueiro, C.A. Salgado, Eur. Phys. J. C 61, (2009) 775

18. B. Schenke, C. Gale, S. Jeon, Phys. Rev. C 80, (2009) 054913

19. T. Renk, arXiv:1009.3740 [hep-ph]

20. T. Sjostrand, S. Mrenna, P. Skands, JHEP 0605, (2006) 026

21. R. Baier, Yu. L. Dokshitzer, A.H. Mueller, D. Schiff, Phys. Rev. C 60, (1999) 064902

22. R. Baier, Yu. L. Dokshitzer, A.H. Mueller, D. Schiff, Phys. Rev. C 64, (2001) 057902

23. Yu.L. Dokshitzer, D. Kharzeev, Phys. Lett. B 519, (2001) 199

24. J.D. Bjorken, Fermilab publication Pub-82/29-THY, 1982

25. E. Braaten, M. Thoma, Phys. Rev. D 44, (1991) 1298

26. I.P. Lokhtin, A.M. Snigirev, Eur. Phys. J. C 16, (2000) 527

27. I.P. Lokhtin, A.M. Snigirev, Phys. Lett. B 440, (1998) 163

28. Yu.L. Dokshitzer, V.A. Khoze, A.H. Mueller, S.I. Troian, Basics of perturbative QCD (Gif-sur-Yvette, France, Ed. Frontieres, 1991)

29. I.P. Lokhtin, L.V Malinina, S.V. Petrushanko, A.M. Snigirev, I. Arsene, K. Tywoniuk, Comput. Phys. Commun. 180, (2009) 779

30. K. Tywoniuk, I.C. Arsene, L. Bravina, A.B. Kaidalov, E. Zabrodin, Phys. Lett. B 657, (2007) 170
31. J.D. Bjorken, Phys. Rev. D 27, (1983) 140

32. M. Cacciari, G.P. Salam, G. Soyez, arXiv:1101.2878 [hep$\mathrm{ph}]$ 